\title{
Propagating solitons generated by localized perturbations on the surface of deep water
}

\author{
H. Xia* and M. Shats \\ Research School of Physics and Engineering, The Australian National University, Canberra, Australian Capital Territory 0200, Australia
}

(Received 7 November 2011; revised manuscript received 17 January 2012; published 16 February 2012)

\begin{abstract}
We report experimental evidence of the propagating capillary solitary waves generated by a localized water surface perturbation. Solitons are formed at the air-water interface with a solid plunger and then propagate with velocities proportional to their amplitudes. The shape of the forward front of such solitons is independent of the plunger shape and is given by the hyperbolic secant profile. We find that the nonlinearity of capillary waves is responsible for a small dispersion in the capillary wave group velocity and the soliton existence. This dispersion can be further reduced by adding minute concentrations of proteins to water.
\end{abstract}

DOI: 10.1103/PhysRevE.85.026313

PACS number(s): 47.35.Fg, 68.03.Kn, 05.45.Yv

\section{INTRODUCTION}

Solitary waves, or solitons, which are spatially localized structures maintaining their shape due to a balance between nonlinearity and dispersion, are found in a broad range of scientific contexts (see, for example, [1]). Despite the fact that a propagating soliton was first observed by Russell in 1834 on the surface of water, there is little experimental evidence of their presence in the deep water surface ripples. The ability of a water surface perturbation to propagate without substantially changing its shape is well understood in shallow layers, described by the Korteweg-de Vries (KdV) equation [2]. In that case, the nonlinearity due to the finite water depth $h$ balances the dispersion of long gravity waves $\omega^{2}=g k \tanh (k h)$. Solitons in optical fibers, which can be generated as a result of modulation instability, were discovered in nonlinear optics under the conditions when the dispersive broadening of a short optical pulse is compensated by selfphase modulation induced by the presence of an intensitydependent refractive index [3-5]

Recently localized oscillating solitons have been found in the water surface ripple under continuous parametric excitation in the capillary-gravity range of frequencies [6]. It has been found that if a water container is shaken in the vertical direction at the frequency $f_{s}$, a parametrically excited standing wave at the first subharmonic frequency $f_{1}=f_{s} / 2$ becomes modulationally unstable and breaks into independent structures oscillating at the frequency $f_{1}$. The surface ripples appear as ensembles of oscillons, or quasiparticles, interacting at short distance. In this case oscillations are constantly forced.

Here we study surface ripples produced by a localized vertically moving plunger. We present experimental evidence that steep capillary waves produced at solid plungers become modulationally unstable and generate solitons at the water-air interface propagating away from the source with the velocity which is proportional to their amplitude. Such solitons had not been observed before on the deep water surface and the mechanism of their generation may clarify the physics of surface oscillations and wake solitons produced by moving solid bodies in contact with water-air interface (e.g., $[7,8]$ ).

All results presented here are obtained when the surface perturbations are generated by a vertically moving $45^{\circ}$ conical plunger seen in Fig. 1. The diameter of the unperturbed

*Hua.Xia@anu.edu.au waterline is $50 \mathrm{~mm}$. Experiments are performed in a square $0.5 \times 0.5 \mathrm{~m}^{2}$ container filled with water to a level of $0.1 \mathrm{~m}$. The plunger is driven in the frequency range between $10 \mathrm{~Hz}$ to several $\mathrm{kHz}$. The forcing is proportional to the acceleration (expressed in units of $g$ ) which is computed as $a_{c}=A \omega_{s}{ }^{2} / g$, where $A$ is the peak-to-peak displacement of the plunger, $g$ is the gravitational acceleration, and $f_{s}=\omega_{s} /(2 \pi)$ is the driving frequency. The gradient of the surface ripple $\nabla \eta$ is measured by reflecting a thin laser beam off the water surface and measuring light intensity $[9,10]$. By integrating in time $\nabla \eta$ of the wave, propagating past the laser reflection point, the surface elevation $\eta$ is deduced. Fast video imaging (up to $600 \mathrm{fps}$ ) is used for the visualization of the fluid surface and for the measurements of the wave-propagation velocities.

\section{SURFACE RIPPLE DEVELOPMENT}

At low acceleration of $a_{c}=3 g$, the surface ripple is represented by the concentric rings shown in Fig. 1(a). As the acceleration is increased to $a_{c}=5 \mathrm{~g}$, the water line of the plunger becomes modulated and the wave fronts become broken, as shown in Fig. 1(b). The breakup of the smooth circular wave front is also observed during the startup stage at higher acceleration. Figure 1(c) shows the time evolution of the surface gradient $\nabla \eta(t)$ measured 150-mm away from the plunger during the startup at $a_{c}=8 g$. The surface gradient which is reasonably symmetric right at the beginning, becomes positively skewed as the instability develops.

We identify three regimes corresponding to three ranges of forcing illustrated in Fig. 2. At low forcing, Figs. 2(a) and 2(d), the plunger produces linear nearly sinusoidal waves. The surface gradient spectrum is characterized by a dominant peak at the forcing frequency $f_{s}=20 \mathrm{~Hz}$. At higher forcing, the water line of the plunger becomes modulated, which is also reflected in the modulation of the wave fronts propagating away from the plunger, Fig. 2(b). This corresponds to the onset of several new features in the frequency spectrum, Fig. 2(e), namely the first subharmonic of the plunger frequency and its multiple harmonics. Further increase in forcing leads to the increase in the surface perturbation steepness, a breaking of the water line into individual "blobs" [clearly seen in Fig. 1(b)] and to the formation of asymmetric wave pulses propagating away from the source. The frequency spectrum at the high forcing level is dominated by the first subharmonic at $f_{1}=10$ Hz, Fig. 2(f). 

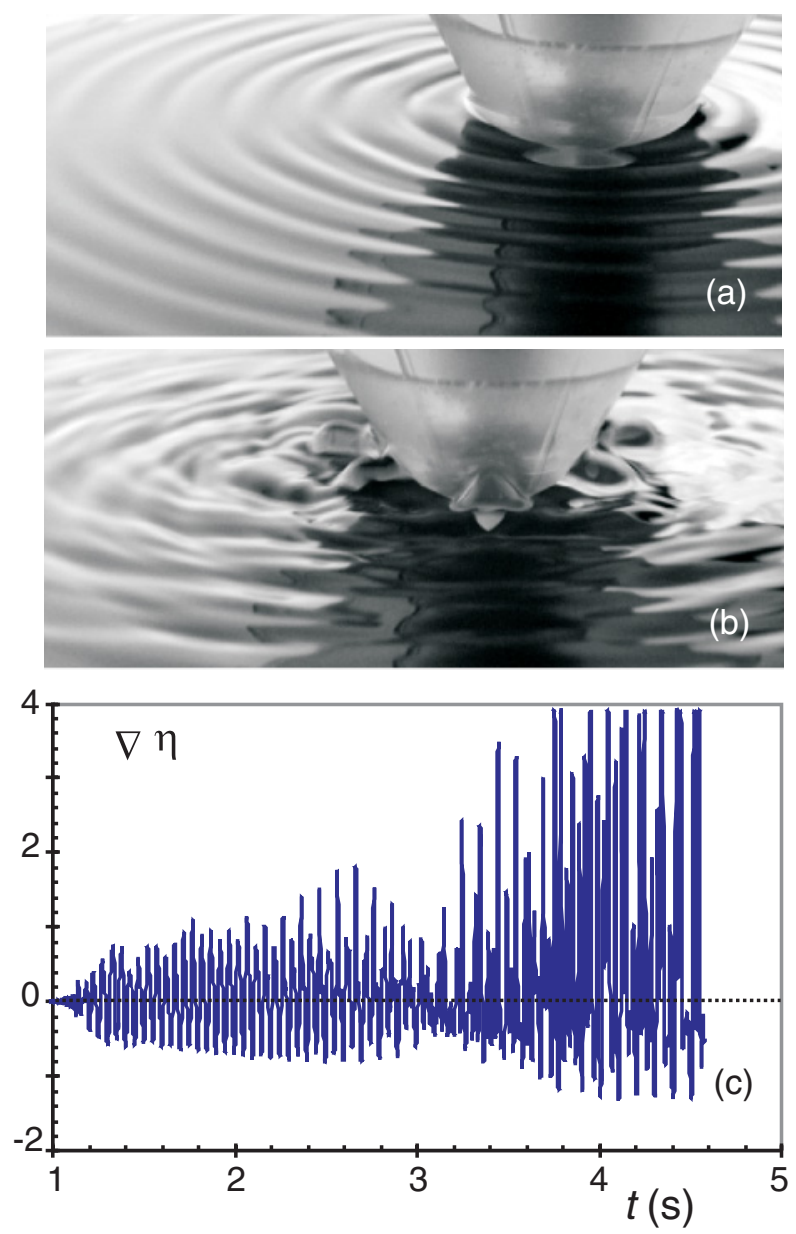

FIG. 1. (Color online) Snapshots of the surface perturbation at different accelerations (a) at $a_{c}=3 g$, (b) $a_{c}=8 g$, and (c) time evolution of the surface gradient at the startup of experiment at $a_{c}=8 g$.

The onset of the subharmonic frequency in the spectrum points to a parametric nature of the instability developing at the plunger. Similar instabilities, known as cross waves, have been studied in rectangular water basins where waves were excited by symmetric wave makers (for review see [11]). The initial azimuthal modulation of the water front is due to the modulation instability of a capillary-gravity wave. It is well known that the gravity surface waves are modulationally unstable, a phenomenon discovered by Benjamin and Feir [12]. Capillary waves are also unstable to small perturbations of the wave envelopes. The instability develops when a Lighthill criterion is satisfied

$$
\left(\partial \omega / \partial|a|^{2}\right)\left(\partial^{2} \omega_{k} / \partial k^{2}\right)<0 .
$$

Here $|a|^{2}$ is the wave intensity, $\omega$ is a nonlinear wave frequency, and $\omega_{k}=\left(T k^{3} / \rho\right)^{1 / 2}$ is a linear dispersion of capillary waves ( $T$ and $\rho$ are the surface tension coefficient and water density, respectively). This criterion states that a wave is unstable if the sign of the group velocity dispersion $\partial^{2} \omega_{k} / \partial k^{2}$ is opposite to $\partial \omega / \partial|a|^{2}$ nonlinearity. An exact nonlinear solution for capillary waves was found by Crapper in 1957 [13], who derived the following expression for the
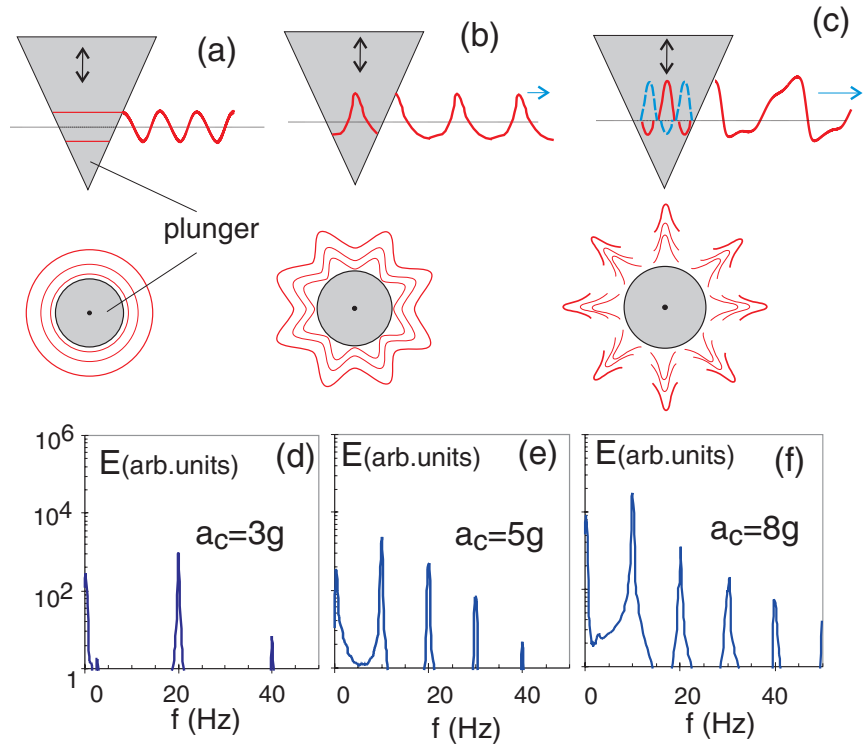

FIG. 2. (Color online) Schematics of the plunger and the underwater view of the surface perturbation at (a) $a_{c}=3 g$, (b) $a_{c}=5 g$, and (c) $a_{c}=8 g$. The corresponding frequency spectra of the surface gradient at (d) $a_{c}=3 g$, (e) $a_{c}=5 g$, and (f) $a_{c}=8 g$. The driving frequency of the plunger is $f_{s}=20 \mathrm{~Hz}$.

capillary wave phase velocity

$$
c=(k T / \rho)^{1 / 2}\left(1+k^{2} a^{2} / 16\right)^{-1 / 4} .
$$

In this expression $a$ is the peak-to-trough amplitude of the wave, as defined in [13]. We follow this definition throughout the remainder of the paper. It is easy to check that the Lighthill criterion (1) is indeed satisfied for capillary waves with $\omega=$ $k c$, where $c$ is given by (2).

The growth rate of the nonlinear stage of the instability leading to the breaking of waves into pulses increases with forcing. Fast video [14] shows that the instability is periodic: the first soliton-like structure is detached from the plunger after about 10 periods of the plunger oscillations. At higher forcing, the instability can develop within a minimum of four plunger periods. Stronger forcing leads to the breaking of the cross wave and ejection of droplets.

A relationship between modulation and parametric instabilities has been established theoretically and numerically [15] and it has been shown that though linear growth rates of the two instabilities are identical, nonlinear stages are different. It should be noted that the development of the modulation instability which leads to the modulation of wave fronts and formation of the oscillating solitons has recently been reported in experiments on parametrically driven surface waves in vertically vibrated containers [6]. The main difference with the results reported here is that in parametrically excited systems standing oscillonic structures are produced which are constantly forced. With the localized excitation, the surface perturbations are formed at the plunger and then propagate away from the source. 

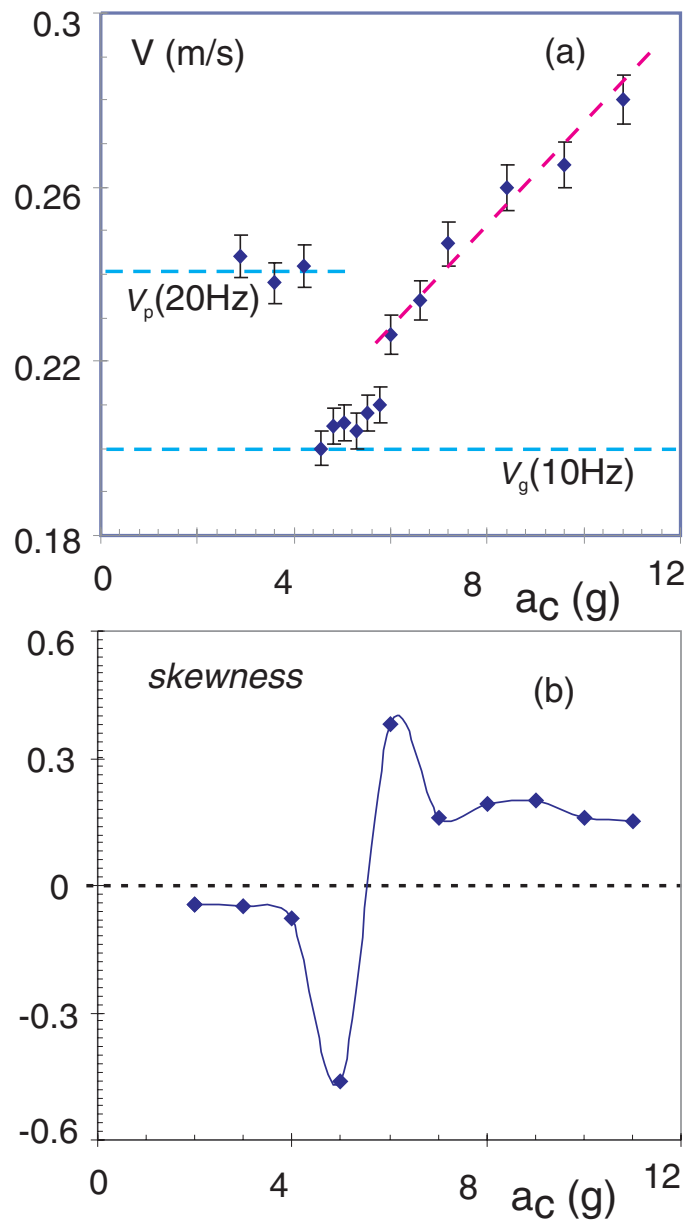

FIG. 3. (Color online) Measurements of (a) the surface perturbation propagating velocity and (b) the skewness of the surface elevation, at different accelerations. The plunger frequency is kept constant at $f_{s}=20 \mathrm{~Hz}$. The acceleration is varied by changing the plunger oscillation amplitude $A$.

\section{SOLITON FORMATION}

Velocities of the surface perturbations were studied using fast video. A perturbation is followed for about $200 \mathrm{~mm}$. The velocities were measured at the distances from the plunger of (20-100) $\mathrm{mm}$ and (100-180) $\mathrm{mm}$. The shape of the waveforms was monitored using laser reflection at the distance $150-\mathrm{mm}$ away from the plunger. The velocity results are presented in Fig. 3(a), while Fig. 3(b) shows the waveform skewness. At low forcing, $a_{c} \leqslant 4 g$, perturbations propagate with velocity which is very close to the capillary wave phase velocity at the frequency of forcing $c\left(f_{s}\right)$. These waves are slightly skewed negatively, as predicted by the nonlinear theory of capillary waves at low amplitudes [13]. Above the threshold of modulation instability, the surface ripples propagate slower, at the speed close to the group velocity of the first subharmonic wave $f_{1}=10 \mathrm{~Hz}$. This regime coincides with the onset of the modulation of the wave fronts. The skewness of the surface ripple changes dramatically and becomes strongly positive. The further increase in forcing at $a_{c}>6 g$ leads to a steady increase in the propagation speed with forcing, Fig. 3(a). The shape of the perturbations, however, does not change in this forcing range, such that the skewness is roughly constant.
This suggests that the propagating structures are solitons which (a) maintain their shape as their propagate, and (b) have velocities which depend on their amplitude. Propagating solitons are subject to viscous dissipation, their amplitudes and velocities are reduced as they propagate away from the plunger. Soliton velocities shown in Fig. 3(a) were measured (20-100) mm away from the plunger. Further away, at (100180) $\mathrm{mm}$, velocities are typically $10-20 \%$ lower. We have also confirmed experimentally that the peak amplitude of the propagating perturbation pulses (solitons) increases linearly with the plunger oscillation amplitude. Thus we conclude that the observed soliton's velocity depends on its amplitude, similar to a $\mathrm{KdV}$ soliton.

Now let us turn to the shape of these solitons. At low amplitude, waves are nearly sinusoidal, as seen in Fig. 4(a). At higher forcing, the surface gradient $\nabla \eta$ consists of pulses which are perfectly fitted by a $D \operatorname{sech}\left(\frac{1}{b} V t\right)$ function, as shown in Fig. 4(b). Here $V$ is the soliton velocity determined from the fast video data, and $b$ is a characteristic pulse width. Hyperbolic secant pulses are well known solutions of the nonlinear Schrödinger equation (e.g., [16]) which are commonly used to describe solitons in various systems, including oscillating oscillons recently found in the parametrically forced capillary ripple [6].

Soliton pulses propagate over large distances (over $80 \mathrm{~mm}$ ) compared with their widths at the nearly constant velocities. The integration of $\nabla \eta$ gives profiles of the surface elevation shown in Fig. 4(d). The shape of these perturbations does not significantly change as they propagate in the near zone, as will be illustrated below. The origin of the $\operatorname{sech}(x)$ pulses in the ripple can be tracked back to the plunger. The cross wave is broken into blobs, as seen in Fig. 4(d). The forward fronts of these blobs are well approximated by the $\operatorname{sech}(x)$ function, as shown in Fig. 4(e). This shape is universal and it is not dependent on the shape of a plunger. We have performed measurements with conical, cylindrical, and triangular prismatic plungers. In all cases the surface gradient signals show ideal sech $(x)$ pulses, as in Fig. 4(b).

The ability of capillary solitons to maintain their shape unchanged can be explained as follows. A capillary-gravity wave dispersion derived from (2) is given by

$$
\omega \approx\left[g k+\left(k^{3} T / \rho\right)\left(1+k^{2} a^{2} / 16\right)^{-1 / 2}\right]^{1 / 2} .
$$

Here we only included nonlinear frequency correction [13] to the capillary branch. Figure 5 shows the dispersion of the group velocity $V_{g}=d \omega / d k$ as a function of wave number $k$ for several amplitudes of waves near the plunger. The surface tension of water is $T=73 \mathrm{mN} / \mathrm{m}$. The $V_{g}(k)$ shows a broad range of $k$ in the capillary waves range, where the nonlinearity balances the dispersion, thus allowing a soliton propagation. The range of the peak-to-trough amplitudes $a$ in Fig. 5 is consistent with the wave amplitudes observed in the vicinity of the plunger [see, e.g., Fig. 4(e)].

\section{PROPAGATION OF SOLITONS}

After solitons are formed as a result of the cross-wave instability at the plunger, they detach from it and propagate in the direction normal to the plunger axis. The number of solitons is determined by the mode number of the cross wave, which 

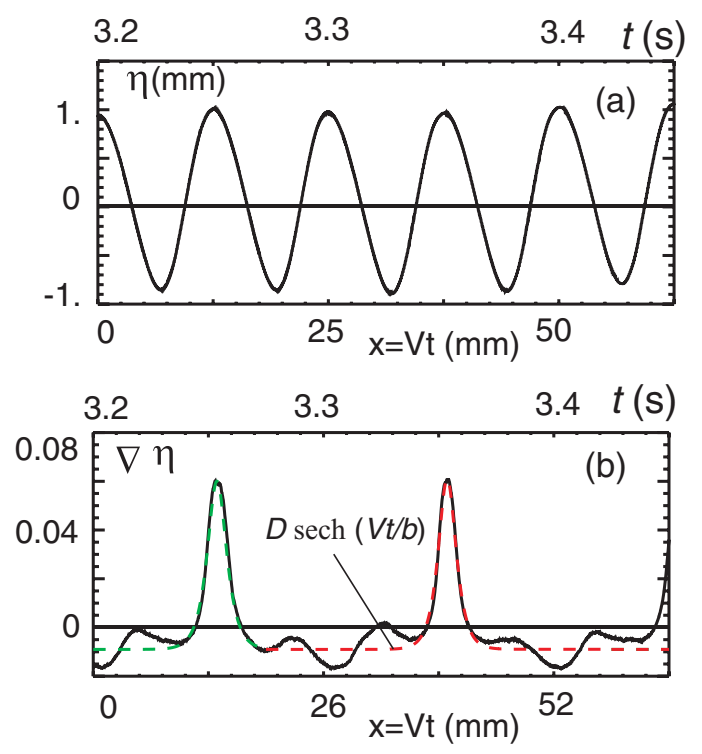

$$
3.2
$$

3.3

$3.4 t(\mathrm{~s})$
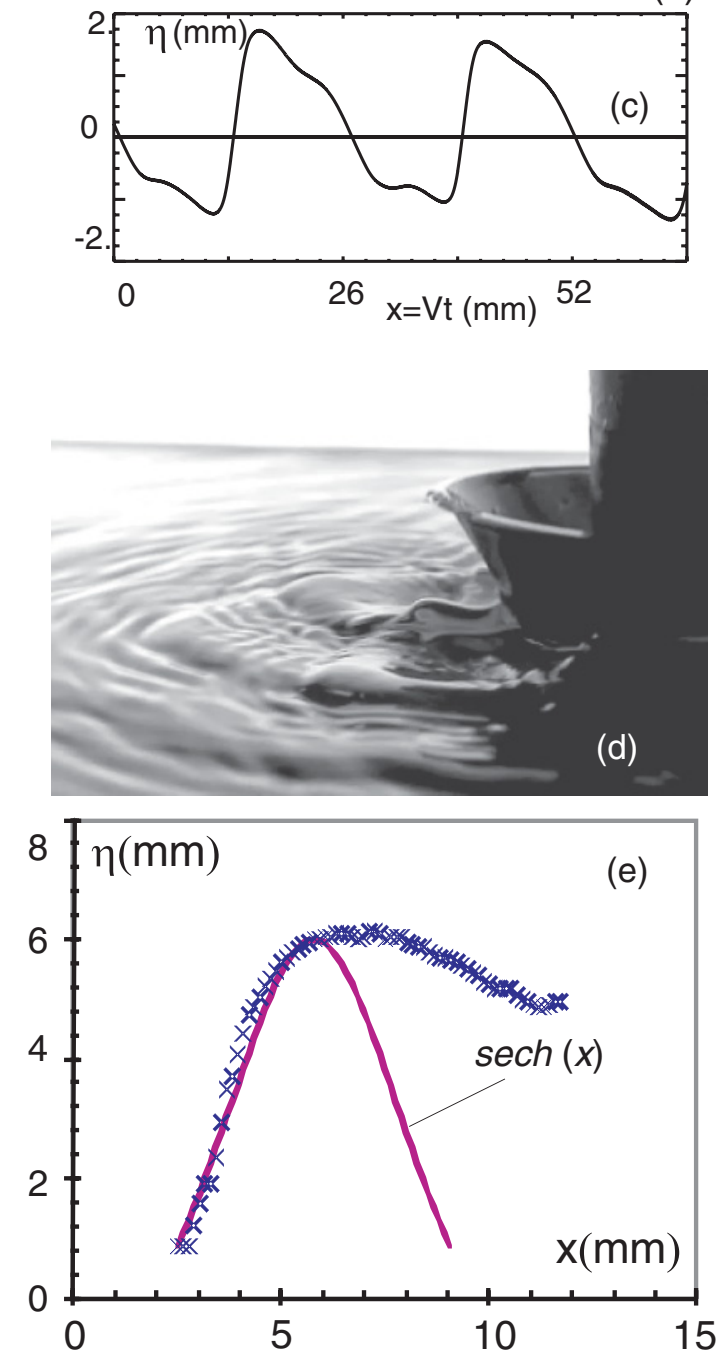

FIG. 4. (Color online) Gradient of surface elevation of the water surface at (a) $a_{c}=3 g$ and (b) $a_{c}=8 g$. (c) Corresponding elevation of the surface at $a_{c}=8 g$. (d) Photo of the soliton generated at the plunger, (e) a waveform of the soliton close to the plunger and its secant hyberbolic fit.

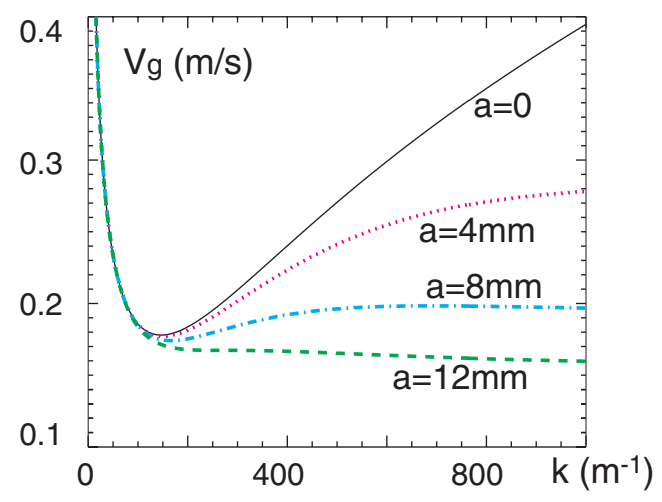

FIG. 5. (Color online) Dispersion of the group velocity based on the capillary wave nonlinear frequency correction at different wave amplitudes.

is a function of both excitation frequency and acceleration. At higher excitation frequencies, solitons are subject to viscous damping which greatly increases with $f$ since damping is proportional to $v k^{2}$, where $v$ is the kinematic viscosity. At lower frequencies, just higher than those corresponding to the minimum of the group velocity, Fig. 5, solitons can propagate longer distances with less damping, however, they gradually disperse, such that the adjacent solitons start overlapping some distance away from the plunger, as illustrated in Fig. 6(a).

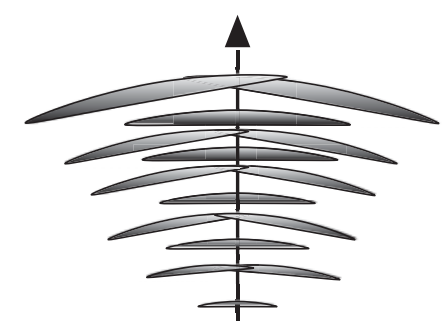

(a)
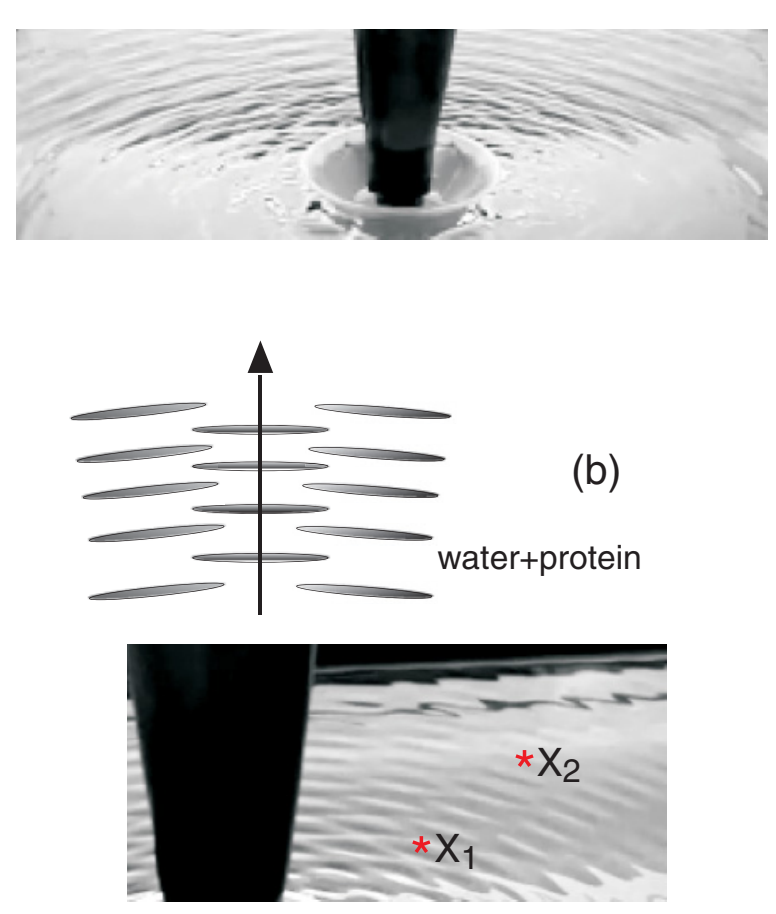

FIG. 6. (Color online) Soliton dispersion in (a) water and (b) in the water suspension of 1 p.p.m. of bovine serum albumin. 

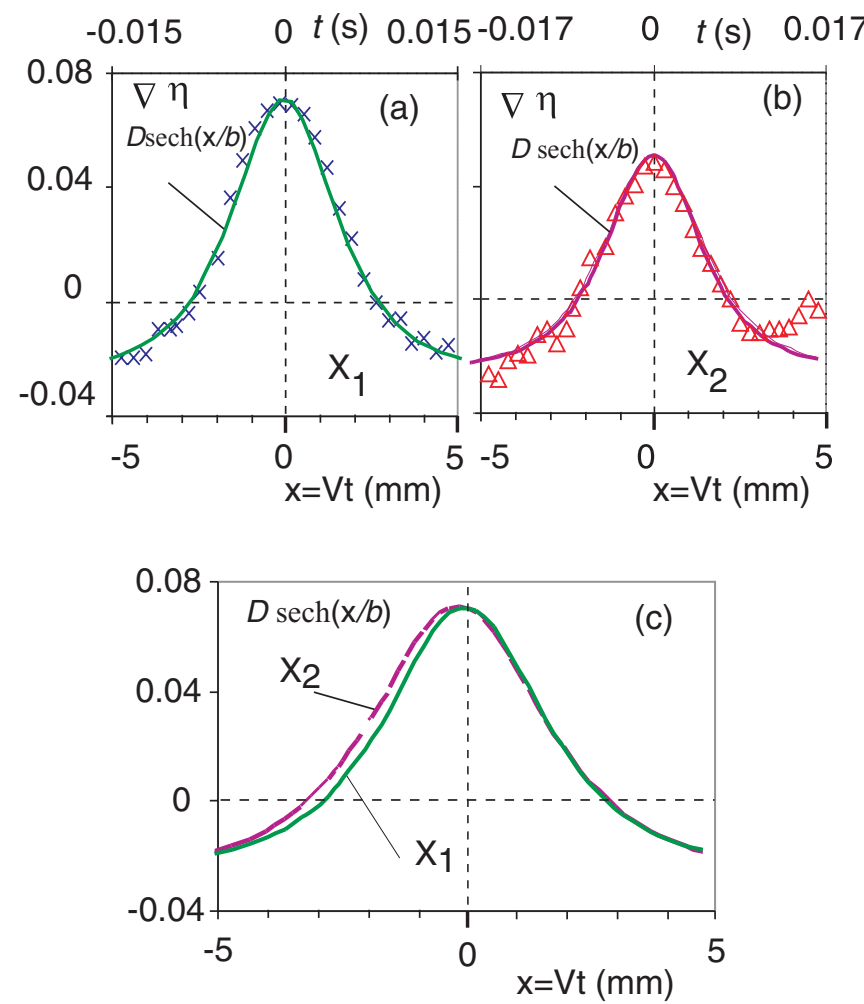

FIG. 7. (Color online) Waveforms of the gradient of the surface elevation measured along the wave propagation at points $X_{1}$ and $X_{2}$ [Fig. 6(b)] located (a) $64 \mathrm{~mm}$ and (b) $94 \mathrm{~mm}$ away from the plunger, respectively. (c) Superimposed normalized sech fits of the waveforms.

A remarkable reduction in the soliton dispersion is observed when microscopic quantities of natural polymers, such as proteins are added into water. This is shown in Fig. 6(b), where 1 p.p.m. (in weight) of bovine serum albumin was added into water. In this case solitons generated at the plunger propagate without dispersion forming nonoverlapping soliton streaks. Though it is not exactly clear how minute concentrations of polymers reduce the dispersion, one can speculate that the modifications of the viscoelastic properties of water affect the capillary wave nonlinearity (3). A rather strong effect of small amounts of proteins added to water has recently been reported in the capillary-gravity ripples under parametric excitation [6]. It has been found that the addition of protein does not noticeably change surface tension or viscosity. More work is needed to understand the effect of natural polymers on surface waves.

To illustrate that the surface perturbations propagate relatively long distances (five to ten times longer then their widths in physical space), we performed measurements of the surface gradient using laser reflection at two points along the wave propagation. These are shown in Fig. 6(b) as points $X_{1}(64$ $\mathrm{mm}$ from the plunger) and $X_{2}$ (94 $\mathrm{mm}$ from the plunger). The corresponding waveforms are shown in Figs. 7(a) and 7(b) along with the sech fitting functions. The shape of both pulses is very similar with the full width at half maximum at point $X_{1}$ of about $3 \mathrm{~mm}$. At point $X_{2}$ the pulse is slightly broader, as seen in Fig. $7(\mathrm{c})$, where the fitting functions are normalized to the same amplitude and superimposed. Thus the sech pulses propagate over the distance of about 10 waveform sizes without changing their shape.

\section{CONCLUSION}

Summarizing, we present new experimental results on the nonlinear generation of solitons on the surface of deep water. Solitons develop at a solid plunger, irrespective of its shape, above certain excitation threshold. Soliton formation occurs via the development of modulation instability of initially linear waves which leads to the cross-wave modulation of the airwater-plunger interface. Then parametrically excited solitons are formed at the plunger at the first subharmonic frequency. Solitons propagate away from the plunger at the velocity which depends on the soliton amplitude. The shape of the forward front of these solitons is universally described by the secant hyperbolic function. A low dispersion of the group velocity derived from the capillary wave nonlinear solution by Crapper [13] supports our finding of the capillary solitary waves.

\section{ACKNOWLEDGMENTS}

The authors are grateful to A. Babanin and H. Punzmann for useful discussions. This work was supported by the Australian Research Council's Discovery Projects funding scheme (DP110101525).
[1] Dissipative Solitons: From Optics to Biology and Medicine, edited by N. Akhmediev and A. Ankiewicz (Springer, Berlin, 2008).

[2] D. J. Korteweg and G. de Vries, Philos. Mag. 39, 422 (1895).

[3] A. Hasegawa and F. Tappert, Appl. Phys. Lett. 23, 142 (1973).

[4] A. Hasegawa, Opt. Lett. 9, 288 (1984).

[5] S. Trillo, S. Wabnitz, E. M. Wright, and G. I. Stegeman, Opt. Lett. 13, 871 (1988).

[6] M. Shats, H. Xia, and H. Punzmann, Phys. Rev. Lett. 108, 034502 (2012).

[7] E. D. Brown, S. B. Buchsbaum, R. E. Hall, J. P. Penhune, K. F. Schmitt, K. M. Watson, and D. C. Wyatt, J. Fluid Mech. 204, 263 (1989).

[8] C. C. Mei and M. Naciri, Proc. R. Soc. London A 432, 535 (1991).
[9] H. Punzmann, M. G. Shats, and H. Xia, Phys. Rev. Lett. 103, 064502 (2009).

[10] M. Shats, H. Punzmann, and H. Xia, Phys. Rev. Lett. 104, 104503 (2010).

[11] J. Miles and D. Henderson, Annu. Rev. Fluid Mech. 22, 143 (1990).

[12] T. B. Benjamin and J. E. Feir, J. Fluid Mech. 27, 417 (1967).

[13] G. D. Crapper, J. Fluid Mech. 2, 532 (1957).

[14] See Supplemental Material at http://link.aps.org/supplemental/ 10.1103/PhysRevE.85.026313 for the development of the crosswave instability during the plunger excitation.

[15] V. I. Karpman, Phys. Lett. A 136, 221 (1989).

[16] V. E. Zakharov and A. M. Rubenchik, Sov. Phys. JETP 38, 494 (1974). 